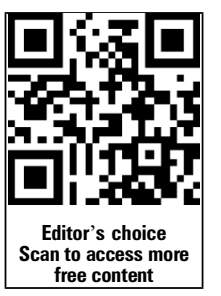

- Additional material is published online only. To view please visit the journal online (http://dx.doi.org/10.1136/ bjsports-2015-094723).

${ }^{1}$ Centre for Sports and Exercise Medicine, Queen Mary University of London, UK ${ }^{2}$ Complete Sports Care, Melbourne, Australia

${ }^{3}$ Pure Sports Medicine, London, UK

${ }^{4}$ Lower Extremity Gait Studies, Health Sciences, La Trobe University, Bundoora, Australia

${ }^{5}$ School of Health and Rehabilitation Sciences, University of Queensland, Brisbance, Queensland, Australia

${ }^{6}$ Physiotherapy Department, Bart's Health NHS Trust, London, UK

\section{Correspondence to} Dr Dylan Morrissey, Centre for Sports and Exercise Medicine, William Harvey Research Institute, Bart's and the London School of Medicine and Dentistry, Queen Mary University of London, Mile End Hospital, Bancroft road, London E1 4DG, UK; d.morrissey@qmul.ac.uk

Accepted 9 June 2015 Published Online First 31 July 2015

\title{
Proximal muscle rehabilitation is effective for patellofemoral pain: a systematic review with meta-analysis
}

\author{
Simon Lack, ${ }^{1}$ Christian Barton, ${ }^{1,2,3,4}$ Oliver Sohan, ${ }^{1}$ Kay Crossley ${ }_{1}^{5}$ Dylan Morrissey ${ }^{1,6}$
}

\begin{abstract}
Background Proximal muscle rehabilitation is commonly prescribed to address muscle strength and function deficits in individuals with patellofemoral pain (PFP). This review (1) evaluates the efficacy of proximal musculature rehabilitation for patients with PFP; (2) compares the efficacy of various rehabilitation protocols; and (3) identifies potential biomechanical mechanisms of effect in order to optimise outcomes from proximal rehabilitation in this problematic patient group.
\end{abstract}

Methods Web of Knowledge, CINAHL, EMBASE and Medline databases were searched in December 2014 for randomised clinical trials and cohort studies evaluating proximal rehabilitation for PFP. Quality assessment was performed by two independent reviewers. Effect size calculations using standard mean differences and 95\% Cls were calculated for each comparison.

Results 14 studies were identified, seven of high quality. Strong evidence indicated proximal combined with quadriceps rehabilitation decreased pain and improved function in the short term, with moderate evidence for medium-term outcomes. Moderate evidence indicated that proximal when compared with quadriceps rehabilitation decreased pain in the short-term and medium-term, and improved function in the medium term. Limited evidence indicated proximal combined with quadriceps rehabilitation decreased pain more than quadriceps rehabilitation in the long term. Very limited short-term mechanistic evidence indicated proximal rehabilitation compared with no intervention decreased pain, improved function, increased isometric hip strength and decreased knee valgum variability while running.

Conclusions A robust body of work shows proximal rehabilitation for PFP should be included in conservative management. Importantly, greater pain reduction and improved function at 1 year highlight the long-term value of proximal combined with quadriceps rehabilitation for PFP.

\section{INTRODUCTION}

Patellofemoral pain (PFP) is one of the most common presentations at both primary care and sports injury clinics. ${ }^{12}$ Prevalence rates in groups of active individuals, including military recruits and novice runners, are reported to be between $3 \%$ and $20 \% .^{3-5}$ PFP has been linked to reduced contact area and increased stress in the lateral patellofemoral joint $(\mathrm{PFJ})^{6}{ }^{7}$ as a result of patellar maltracking, including greater lateral patellar translation, ${ }^{8-10}$ tilt $^{8}$ and spin. ${ }^{9}$ The cause of maltracking in PFP is thought to be multifactorial with local, ${ }^{11}$ distal $^{12}$ and proximal ${ }^{13}$ factors proposed to contribute to it, with good evidence that long axis femoral rotation in relation to the patella is a key contributor to maltracking and a valid rehabilitation target. ${ }^{14}$

Consistent with the multifactorial nature of PFP, management of PFP has traditionally focused on a variety of interventions, including rest, analgesia, general quadriceps and vastus medialis oblique rehabilitation exercises, proximal rehabilitation exercises, patellar taping, foot orthoses and gait retraining. ${ }^{15}$ Each of these interventions has a varying level of efficacy, with multimodal interventions appearing to be the most effective. ${ }^{16}{ }^{17}$ Growing evidence for impaired proximal muscle strength ${ }^{18-20}$ and function, ${ }^{21}$ combined with links between hip mechanics and increased risk of PFP, ${ }^{52}$ has resulted in promotion of rehabilitation aimed at addressing impairments in proximal musculature. ${ }^{14}$ Our recent mixed methods study of international experts' clinical reasoning when managing PFP supported this recommendation, but a lack of supporting level-one evidence was also identified. ${ }^{17}$ The effectiveness of proximal rehabilitation protocols have been evaluated in high quality recent research, ${ }^{23-26}$ and commonly consist of open and closed kinetic chain exercises which reflect clinical practice. ${ }^{27}$

A recent low quality (LQ) systematic review concerning proximal rehabilitation for $\mathrm{PFP}^{28}$ concluded that hip interventions were effective in improving pain and function in individuals with PFP. However, the search for available evidence was limited to a 2-year period (January 2011-January 2013), with no attempt at data pooling nor mechanistic exploration and there is, therefore, a need for a more detailed and inclusive review in order to optimally guide practice.

Our systematic review and meta-analysis aims to (1) evaluate the effects of proximal muscle rehabilitation for patients with PFP, (2) compare the effects of various rehabilitation protocols, and (3) evaluate potential mechanism of action in order to optimally guide clinical practice in rehabilitating patients. Further, we aimed to promote clarity in rehabilitation programme design and reporting, with particular respect to the term 'strengthening'.

\section{METHODS}

The PRISMA statement was consulted prior to the start of this review and the checklist completed. ${ }^{29}$

\section{Inclusion and exclusion criteria}

Randomised clinical trials (RCTs) and cohort studies evaluating proximal muscle rehabilitation programmes were considered for inclusion. A 
proximal muscle rehabilitation protocol was defined as progressive exercise directed at the hip or lumbopelvic musculature or both. Studies of multimodal interventions were included where the effects of adding proximal rehabilitation could be clearly determined. Case reports and non-English studies were excluded. The inclusion criteria required participants to be described as having PFP, anterior knee pain or chondromalacia patella in the absence of other knee pathologies, including patellar tendinopathy, Osgood-Schlatters disease and SindingLarsen-Johanssons syndrome. Studies evaluating all age ranges were considered for inclusion, as well as studies involving both single sex and mixed sex sample groups.

\section{Search strategy}

Web of Knowledge, CINAHL, EMBASE and Medline (via OVID) databases were searched from inception to December 2014 , using the search strategy outlined in box 1. Reference lists of included publications were screened and citation tracking was completed in Google Scholar.

\section{Review process}

Titles and abstracts identified using the search strategy were downloaded into EndNote X7.1 (Thomson Reuters, California, USA). Duplicates were deleted before all abstracts were screened for inclusion by two independent reviewers (SL and OS). A third reviewer (CB) was available to settle any disputes if necessary. Full texts were obtained where necessary.

\section{Quality assessment}

Study methodological quality was assessed with the PEDro scale $^{30}$ and a PFP inclusion/exclusion criteria checklist ${ }^{31}$ by two independent reviewers (OS and SL). Discrepancies were resolved by consensus, with a third reviewer (CB) available, if needed. Based on the PEDro scores, ${ }^{30}$ and guidance by Moher et al, ${ }^{32}$ studies scoring $>6$ were considered high quality (HQ) and $\leq 6$ as LQ. The PFP diagnosis checklist ${ }^{31}$ is a seven-item scale that identifies key inclusion and exclusion criteria for the diagnosis of PFP. Higher scores indicate a greater number of key criteria having been reported.

\section{Study analysis}

Sample sizes, participant demographics, interventions, variables evaluated and follow-up times were extracted from each study. Further analysis of intervention programme design was completed to determine the type of 'rehabilitation' that was prescribed (table 2) allowing for direct comparison between

\section{Box 1 Search strategy}

Patellofemoral Pain OR Anterior Knee Pain OR Patellofemoral Syndrome OR retropatellar pain OR peripatellar pain OR patellofemoral joint pain OR parapatellar pain OR PFP OR chondromalacia patellae

\section{AND}

Proximal OR gluteal

AND

Strength*

AND

Training OR program OR exercise OR rehab*

*, a truncation indicator for searching. reported training methodology and accepted principles of 'neuromuscular activation' (exercise performed at $<30 \% 1$ repetition maximum, for $>20$ repetitions), 'strength' (exercise performed $\geq 70 \% 1$ repetition maximum), 'strength-endurance' (exercise performed at $30-70 \% 1$ repetition maximum) and 'power' (exercise performed at either $85-100 \% 1$ repetition maximum or $0-60 \% 1$ repetition maximum at an explosive velocity). ${ }^{33}$ This was in response to concerns raised at the recent PFP research retreat in Vancouver ${ }^{15}$ that the word strengthening or strength training is used synonymously for all types of rehabilitation exercise consequently limiting the identification of exercise prescription specificity. It was considered that evaluating methods of exercise prescription (eg, focus on strength, endurance, etc.) and summarising the range of specific exercise descriptors ( $\%$ repetition maximum, repetitions, time-undertension) could maximise the clinical utility of this review and facilitate translation to clinical practice.

Means and SDs for all baseline and follow-up data were extracted and entered into Cochrane Review Manager (V.5.2) to allow calculation of standard mean differences (SMDs). Meta-analysis was completed where studies evaluated similar interventions using comparable outcome measures (eg, VAS, Visual Analogue Scale and NPRS, Numeric Pain Rating Scale). Where multiple measures were used, a consistent measure between studies was used for pooling (eg, stair ascent). Pooling of data across time points was performed for studies that evaluated outcomes in the 'short term' ( $<3$ months), 'medium term' (3-12 months), and 'longer term' ( $\geq 12$ months). All outcome measure scores were converted so that favourable outcomes (reduced pain, improved function, improved strength, etc) were entered as positive values into Cochrane Review Manager Software, to facilitate consistent visual representation of SMDs and pooled findings along with 95\% CIs. For studies without comparative groups, the results were extracted and reported in the results section, but no meta-analysis performed. Following methodology proposed by Hume et $a l,{ }^{34}$ individual and pooled SMDs were categorised as small $(\leq 0.59)$, medium $(0.60-1.19)$, or large $(\geq 1.20)$. These criteria were chosen to increase stringency compared to traditional criteria. ${ }^{35}$ Statistical heterogeneity of pooled data was established using the $\mathrm{X}^{2}$ and $\mathrm{I}^{2}$ statistics (with heterogeneity being defined as $\mathrm{p}<0.05$ ). Levels of evidence were guided by recommendations proposed by Van Tulder et al: ${ }^{36}$

Strong evidence $=$ based on results derived from multiple studies, including a minimum of two HQ studies, which are statistically homogenous $\left(\mathrm{I}^{2}<50 \%\right)$.

Moderate evidence $=$ based on results derived from multiple studies, including at least one HQ study, which are statistically heterogeneous $\left(\mathrm{I}^{2}>50 \%\right)$, or from multiple LQ studies which are statistically homogenous $\left(\mathrm{I}^{2}<50 \%\right)$.

Limited evidence $=$ based on results derived from multiple LQ studies which are statistically heterogeneous $\left(\mathrm{I}^{2}>50 \%\right)$, or from one HQ study.

Very limited evidence $=$ based on results derived from one LQ study.

Conflicting evidence=based on insignificant pooled results derived from multiple studies regardless of quality, which are statistically heterogeneous $\left(\mathrm{I}^{2}>50 \%\right)$.

\section{RESULTS}

The results of the database search are shown in figure 1. Fourteen studies were identified for the final review. Eleven of these studies were randomised or comparative control trials, ${ }^{23} 242637-44$ and three were cohort studies. ${ }^{25} 4546$ Study details, including sample sizes and participant demographics, are shown in online 
Table 1 PEDro scale

\begin{tabular}{|c|c|c|c|c|c|c|c|c|c|c|c|c|}
\hline Author & I & II & III & IV & V & VI & VII & VIII & IX & $x$ & XI & Total score \\
\hline Baldon et $a l^{44}$ & 1 & 1 & 1 & 1 & 1 & 0 & 1 & 1 & 1 & 1 & 1 & 10 \\
\hline Nakagawa et al ${ }^{40}$ & 0 & 1 & 1 & 1 & 1 & 0 & 1 & 1 & 1 & 1 & 1 & 9 \\
\hline Ismail et $\left.a\right|^{39}$ & 1 & 1 & 1 & 1 & 0 & 0 & 0 & 1 & 1 & 1 & 1 & 8 \\
\hline Fukuda et $a l^{23}$ & 1 & 1 & 1 & 1 & 0 & 0 & 1 & 1 & 1 & 1 & 1 & 8 \\
\hline Fukuda et $a l^{38}$ & 1 & 1 & 1 & 1 & 0 & 0 & 1 & 1 & 1 & 1 & 1 & 8 \\
\hline Ferber et $a l^{42}$ & 1 & 1 & 1 & 0 & 0 & 0 & 1 & 0 & 1 & 1 & 1 & 7 \\
\hline Khayambashi et $a l^{43}$ & 1 & 1 & 0 & 1 & 0 & 0 & 0 & 1 & 1 & 1 & 1 & 7 \\
\hline Razeghi et $a l^{37}$ & 1 & 1 & 1 & 0 & 0 & 0 & 0 & 1 & 1 & 1 & 1 & 6 \\
\hline Dolak et $a l^{24}$ & 0 & 1 & 1 & 1 & 0 & 0 & 0 & 0 & 1 & 1 & 1 & 6 \\
\hline Ferber et $a l^{46}$ & 1 & 0 & 0 & 1 & 0 & 0 & 0 & 1 & 1 & 1 & 1 & 5 \\
\hline Khayambashi et al ${ }^{26}$ & 0 & 0 & 0 & 1 & 0 & 0 & 0 & 1 & 1 & 1 & 1 & 5 \\
\hline Avraham et $a l^{41}$ & 0 & 1 & 0 & 0 & 0 & 0 & 1 & 0 & 1 & 1 & 0 & 4 \\
\hline Tyler et $a l^{45}$ & 1 & 0 & 0 & 0 & 0 & 0 & 0 & 1 & 1 & 0 & 1 & 3 \\
\hline Earl and $\mathrm{Hoch}^{25}$ & 1 & 0 & 0 & 0 & 0 & 0 & 0 & 1 & 1 & 0 & 1 & 3 \\
\hline
\end{tabular}

supplementary file 1 . Intervention and control/comparison group protocols, outcome measures and follow-up duration findings are presented in online supplementary files $2-5$.

\section{Quality assessment}

Results from the PFP diagnostic checklist and the PEDro scale are shown in online supplementary file 6 and table 1, respectively. All 12 studies scored five or greater out of seven on the PFP diagnostic checklist, demonstrating a good level of consistency between studies for diagnostic inclusion/exclusion criteria. Scores ranged between 3 and 10 for the PEDro scale. Of the 14 studies, 7 were classed as HQ, ${ }^{23}{ }^{38-40} 42-44$ and 7 were classed as LQ. ${ }^{24-2637414546}$

\section{Exercise prescription and mechanobiological analysis}

Results of exercise prescription and mechanobiological analysis are shown in tables 2 and 3, respectively. Three of the 14 included studies 233844 were considered to have evaluated the same exercise approach as stated in their title and methodology. Commonly (10 of 14 studies), studies reported evaluation of a strength protocol, despite exercise programmes being considered to be of an intensity to evoke strengthendurance $^{243739424546}$ or neuromuscular ${ }^{25264043}$ activation changes. In one study, the description of exercise prescribed was unclear and could not be interpreted. ${ }^{41}$ Analysis of mechanobiological descriptors of exercise prescription from within the 14 included studies highlighted the absence of all 'classical descriptors' (eg, load magnitude, \% of maximum) and rest period between sets $(\mathrm{s} / \mathrm{min}))$ in all but one study ${ }^{39}$ (table 3$)$. The seven new descriptors proposed by Toigo and Boutellier ${ }^{47}$ were absent, in their entirety, in the methodology of all included studies. Inclusion of the 13 descriptors in future studies is reported to be imperative for the delivery of effective and tailored exercise prescription. ${ }^{47}$

\section{Effects of proximal rehabilitation}

Proximal rehabilitation-compared with—control

\section{Pain and function}

One LQ study ${ }^{26}$ compared proximal rehabilitation in PFP patients to a control group receiving only Omega-3 and calcium supplementation in the short term (figure 2). Very limited evidence (1 LQ study $^{26}$ ) with large effect indicated proximal rehabilitation, using exclusively open kinetic chain (OKC) exercises with progressively higher resistant elastic band (see online supplementary file 2 for further programme details), reduces pain (VAS) (SMD, 95\% CI 2.80,1.71 to 3.88), and improves function (Western Ontario and McMaster osteoarthritis index (WOMAC)) (SMD, 95\% CI 2.88, 1.78 to 3.98) in the short term.

Proximal rehabilitation-compared with—quadriceps rehabilitation

Pain

Three $\mathrm{HQ}^{42-44}$ and one LQ study ${ }^{24}$ compared proximal rehabilitation to quadriceps rehabilitation in the short and medium term (see online supplementary file 3 for further programme details; figure 3). Moderate evidence ( $3 \mathrm{HQ}^{42-44}$ and $1 \mathrm{LQ}^{24}$ study) of a small effect indicated greater pain reduction following a proximal rehabilitation programme compared with a quadriceps rehabilitation protocol in the short term $\left(\mathrm{I}^{2}=81 \%, \mathrm{p}=0.001\right.$; SMD, 95\% CI $0.36,0.13$ to 0.59 ). In the medium term, there was strong evidence ( 2 HQ studies ${ }^{43}$ ) of a medium effect indicating greater pain reduction following a proximal rehabilitation programme compared with a quadriceps rehabilitation protocol $\left(\mathrm{I}^{2}=45 \%, \mathrm{p}=0.18\right.$; SMD, 95\% CI $1.07,0.55$ to 1.59$)$.

\section{Function}

Moderate evidence ( $3 \mathrm{HQ}^{42-44}$ and $1 \mathrm{LQ}^{24}$ study) indicated no difference in functional patient-reported outcome measures (Lower Extremity Functional Scale, LEFS; Anterior Knee Pain Score; AKPS and WOMAC) within a pooled group of statistically heterogeneous studies comparing proximal and quadriceps rehabilitation protocols in the short term $\left(\mathrm{I}^{2}=69 \%, \mathrm{p}=0.02\right.$; SMD, 95\% CI $0.18,-0.05$ to 0.42 ). In the medium term, strong evidence (2 HQ studies ${ }^{43}$ ) of medium effect indicated proximal rehabilitation improves functional patient-reported outcome measures (LEFS and WOMAC) when compared with quadriceps rehabilitation protocols $\left(\mathrm{I}^{2}=0 \%, \mathrm{p}=0.54 ; \mathrm{SMD}\right.$, 95\% CI $0.87,0.36$ to 1.37 ). Limited evidence (1 HQ study ${ }^{44}$ ) of a medium effect indicated improved objective function, as measured by single leg hop performance, following proximal compared to quadriceps rehabilitation in the short term.

Proximal combined with quadriceps rehabilitation-compared with-quadriceps rehabilitation

Four $\mathrm{HQ}^{23}{ }^{38-40}$ and three $\mathrm{LQ}^{24} 37^{41}$ studies compared proximal combined with quadriceps rehabilitation to quadriceps 
Table 2 Analysis of programme design and aims

\begin{tabular}{|c|c|c|c|c|c|c|}
\hline Author & $\begin{array}{l}\text { Stated exercise } \\
\text { aim (title) }\end{array}$ & $\begin{array}{l}\text { Method exercise aim } \\
\text { (within text) }\end{array}$ & $\begin{array}{l}\text { Reviewers' interpretation of } \\
\text { actual exercise aim }\end{array}$ & $\begin{array}{l}\text { Fit of stated exercise aim (in text) } \\
\text { and actual exercise }\end{array}$ & Outcome & measure suitability for reviewer defined exercise aim and comment \\
\hline Nakagawa et al ${ }^{40}$ & Str & Str & NM & 0 & 1 & $\begin{array}{l}\text { No patient capacity specific loading; no additional load; Assessed muscle } \\
\text { EMG and isokinetic strength }\end{array}$ \\
\hline Ismail et $\left.a\right|^{39}$ & Str & Str & StrEnd & 1 & 2 & $\begin{array}{l}\text { Proximal rehabilitation performed at } 60 \% \text { of } 10 \text { RM; No patient specific } \\
\text { training intensity for CKC exercises reported }\end{array}$ \\
\hline Fukuda et $a l^{23}$ & Str & Str & Str & 2 & 1 & $\begin{array}{l}\text { Programme used } 70 \% \text { of 'estimated } 1 \text { RM' that could be performed pain } \\
\text { free }\end{array}$ \\
\hline Fukuda et $a^{\beta 8}$ & Str & Str & Str & 2 & 1 & $\begin{array}{l}\text { Programme used } 70 \% \text { of 'estimated } 1 \text { RM' that could be performed pain } \\
\text { free }\end{array}$ \\
\hline Razeghi et a $\left.\right|^{37}$ & Str & Str & StrEnd & 1 & 2 & McQueen method of load progression used \\
\hline Dolak et $a l^{24}$ & Str & Str & StrEnd & 1 & 2 & Load progression as $\%$ of body weight, not of muscle capacity \\
\hline Ferber et $a l^{46}$ & Str & Str & StrEnd & 1 & 1 & $\begin{array}{l}\text { Progression offered if exercise performed 'too easily', definition of 'too } \\
\text { easily' not reported }\end{array}$ \\
\hline Ferber et $a l^{42}$ & Str & NM & StrEnd & 1 & 1 & $\begin{array}{l}\text { Clinician led load prescription, ensuring last } 3 \text { of } 10 \text { repetitions were } \\
\text { 'challenging' }\end{array}$ \\
\hline $\begin{array}{l}\text { Khayambashi } \\
\text { et } a l^{26}\end{array}$ & Str & Str & NM & 0 & 2 & $\begin{array}{l}\text { Resistance progression was generic, } 3 \times 20-25 \text { repetitions were performed } \\
\text { for each exercise }\end{array}$ \\
\hline Baldon et a ${ }^{44}$ & $\mathrm{NM} / \mathrm{Str}$ & $\mathrm{NM} / \mathrm{Str}$ & $\mathrm{NM} / \mathrm{Str}$ & 2 & 2 & $\begin{array}{l}\text { NM programme } 20 \text { repetitions plus isometric holds; Str performed at } 75 \% \\
1 \text { RM } 3 \times 12 \text { repetitions }\end{array}$ \\
\hline $\begin{array}{l}\text { Khayambashi } \\
\text { et a } /^{43}\end{array}$ & Str & Str & NM & 0 & 1 & $\begin{array}{l}\text { Generic progression of resistance, } 3 \times 20-25 \text { repetitions were performed } \\
\text { for each exercise }\end{array}$ \\
\hline Avraham et a ${ }^{41}$ & Str & Str & Unclear & Unclear & Unclear & $\begin{array}{l}\text { Programme not clearly defined, repetitions dependent on patient } \\
\text { 'capability'; no additional load described }\end{array}$ \\
\hline Tyler et $a l^{45}$ & Not stated & Str & StrEnd/NM/P & 0 & 1 & $\begin{array}{l}\text { Programme described as 'progressive resistive exercise' but exact number } \\
\text { of repetitions and load not described }\end{array}$ \\
\hline Earl and $\mathrm{Hoch}^{25}$ & Str & NM & NM & 1 & 1 & Assessed kinematic change, but also assessed strength \\
\hline
\end{tabular}

$0=$ No, $1=$ In part, $2=$ Yes. (30-70\% 1 RM). 


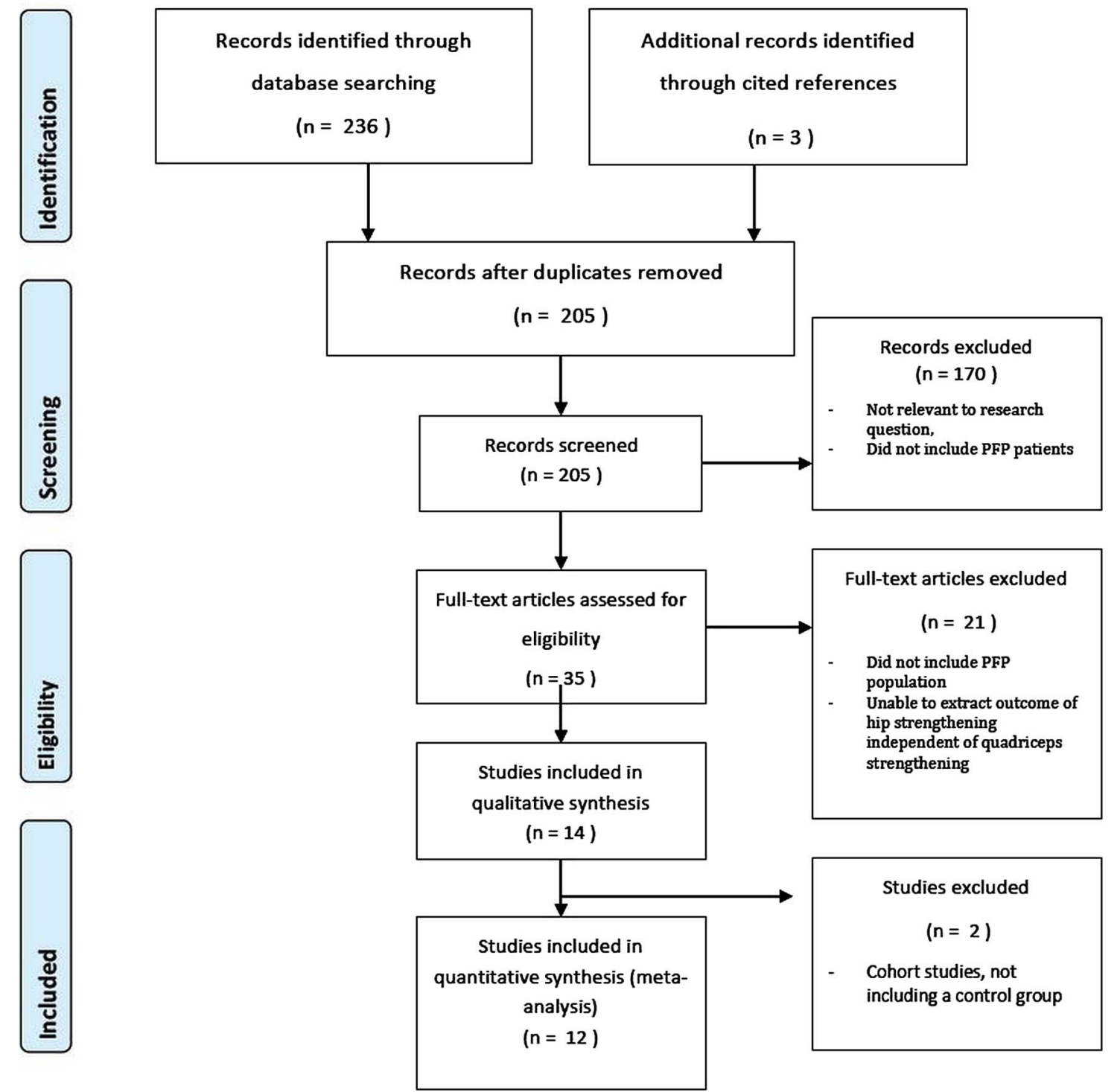

Figure 1 Flow chart of included studies. PFP, patellofemoral pain.

rehabilitation alone (see online supplementary file 4 for further programme details), covering short, ${ }^{23} 24 \quad 37 \quad 39-41 \quad 46$ medium, ${ }^{23} 24$ and longer ${ }^{23}$ follow-up (figures 4 and 5).

\section{Pain}

Strong evidence $\left(3 \mathrm{HQ}^{38-40}\right.$ and $2 \mathrm{LQ}$ studies ${ }^{24}$ ) of a small effect indicated greater pain reduction favouring proximal combined with quadriceps rehabilitation using both OKC and CKC exercises compared to a quadriceps rehabilitation alone in the short term $\left(\mathrm{I}^{2}=14 \%, \mathrm{p}=0.33\right.$; SMD, 95\% CI $0.55,0.22$ to $0.88)$. In the medium term, there was moderate evidence $\left(1 \mathrm{HQ}^{23}\right.$ and $\left.1 \mathrm{LQ} \mathrm{study}^{24}\right)$ of a large effect indicating greater pain reduction following proximal combined with quadriceps rehabilitation using $\mathrm{OKC}$ and $\mathrm{CKC}$ compared to quadriceps rehabilitation alone in the medium term $\left(\mathrm{I}^{2}=92 \%, \mathrm{p}=0.0003\right.$; SMD, $95 \%$ CI $1.36,0.83$ to 1.90$)$. Within the same HQ study, at 6 months, there was limited evidence ( $1 \mathrm{HQ}$ study $^{23}$ ) of a large effect indicating greater reduction in pain following proximal combined with quadriceps rehabilitation compared to quadriceps rehabilitation alone (SMD, 95\% CI 2.58, 1.81 to 3.35).

In the longer term, there was limited evidence (1 HQ study $^{23}$ ) of a large effect indicating greater pain reduction following proximal combined with quadriceps rehabilitation compared to quadriceps rehabilitation alone (SMD, 95\% CI $2.99,2.16$ to 3.83 ).

\section{Function}

Strong evidence $\left(2 \mathrm{HQ}^{38} 39\right.$ and $1 \mathrm{LQ}^{24}$ study) of a small effect indicated greater functional patient-reported improvement (how measured) following proximal combined with quadriceps rehabilitation compared to quadriceps rehabilitation alone in the short term $\left(\mathrm{I}^{2}=18 \%, \mathrm{p}=0.30 ; \mathrm{SMD}, 95 \% \mathrm{CI} 0.42,0.03\right.$ to 0.81 ). Limited evidence (1 HQ study ${ }^{38}$ ) indicated no difference in functional performance measured with the single leg hop test scores (SMD, 95\% CI $0.32,-0.30$ to 0.93 ) in the short term.

In the medium term, moderate evidence $\left(1 \mathrm{HQ}^{23}\right.$ and $1 \mathrm{LQ}^{24}$ study) of a large effect indicated increased patient reported function, measured by LEFS $\left(\mathrm{I}^{2}=96 \%, \mathrm{p}<0.00001\right.$; SMD, 95\% CI $1.32,0.75$ to 1.89 ). Limited evidence (1 HQ study ${ }^{23}$ ) of a large effect indicated increased patient-reported function measured by AKPS (SMD, 95\% CI 1.86, 1.18 to 2.54). Limited evidence (1 HQ study ${ }^{23}$ ) of large effect indicates increased performance-based function, measured by single leg hop scores (SMD, 95\% CI 1.54, 0.89 to 2.18). Within the same study, at 
Table 3 Analysis of specific descriptors of exercise prescription Classical set of descriptors

\section{New set of descriptors}

\section{Fractional and}

temporal

distribution of the

$\begin{array}{lll}\text { exercise } & \text { Duration of the } & \text { contraction modes } \\ \text { interventions } & \text { experimental } & \text { per repetition and } \\ \text { (per [day] or } & \text { period ([day] or } & \text { duration [s] of one }\end{array}$

$\begin{array}{lll}\text { exercise } & \text { Duration of the } & \text { contraction modes } \\ \text { interventions } & \text { experimental } & \text { per repetition and } \\ \text { (per [day] or } & \text { period ([day] or } & \text { duration [s] of one }\end{array}$

Rest Time

under

Recovery time
in-between

in-between

tension

Anatomical repetition ([s] or [min])

([s] or
[min])

muscular

Range
of

sessions ([h]

weeks)

$x \quad x$

failure

motion [d])

\begin{tabular}{lllll}
\hline Nakagawa & $X$ & $Y$ & $Y$ & $X$
\end{tabular}

Y Y

$\mathrm{x}$

$\mathrm{x}$

$\mathrm{x}$

$x$

definition of

et al ${ }^{40}$

Ismail et $a^{39} \quad Y$

Fukuda et $a l^{23} \quad Y$

Fukuda et $a l^{38} \quad \mathrm{Y}$

Razeghi

et $a l^{\beta 7}$

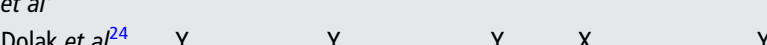

Dolak et $a l^{24}$

Ferber et a ${ }^{42} \quad X$

Khayambashi $X$

et $a l^{26}$

Baldon et a ${ }^{44} \quad \mathrm{Y}$

Khayambashi $X$

et $a l^{43}$

Avraham $\mathrm{x}$

et $a l^{41}$

Tyler et $a l^{45} \quad \mathrm{X}$

Earl and

$\mathrm{Y}=$ Incorporated in study, $\mathrm{X}=$ Not incorporated in study.

$Y$
$X$
$X$
$X$
$X$
$Y$
$X$
$X$
$X$
$X$
$X$
$X$
$X$

$X$
$X$
$X$
$X$
$X$
$X$
$X$
$X$
$X$
$X$
$Y$
$X$
$X$

$\begin{array}{ll}x & x \\ x & x \\ x & x \\ x & x\end{array}$

$X \quad Y$

(exercise form) 


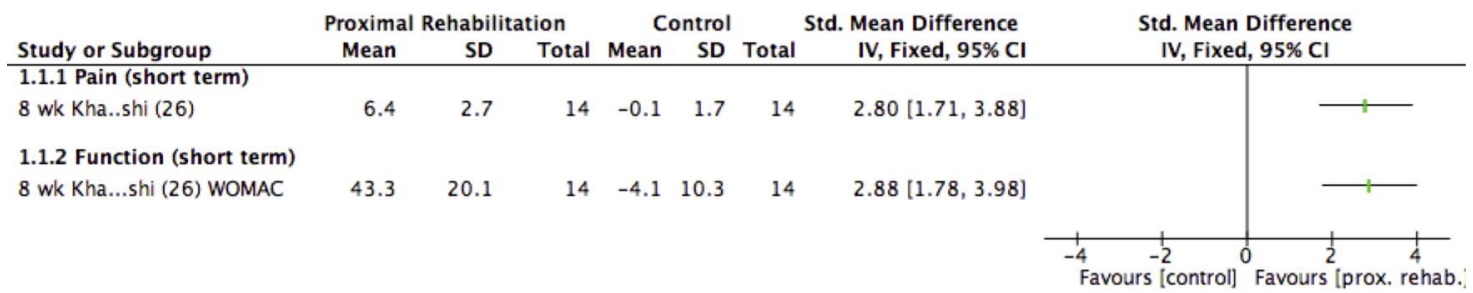

Figure 2 Proximal rehabilitation compared with control group for pain and function (WOMAC, Western Ontario and McMaster osteoarthritis index; 'short term', <3 months; IV, inverse variance; Std., standard mean difference).

6 months, limited evidence ( 1 HQ study ${ }^{23}$ ) of a large effect indicated increased patient-reported function, as measured by LEFS (SMD, 95\% CI 2.49, 1.73 to 3.25), AKPS (SMD, 95\% CI $1.86,1.18$ to 2.54 ), and performance-based function measured as single leg hop scores (SMD, 95\% CI 1.85, 1.17 to 2.52 ).

In the longer term, limited evidence ( $1 \mathrm{HQ} \operatorname{study}^{23}$ ) of a large effect indicated greater patient-reported functional improvement as measured by LEFS (SMD, 95\% CI 2.65, 1.86 to 3.43 ), AKPS (SMD, 95\% CI 1.76, 1.09 to 2.42 ), and performance-based function measured with the single leg hop (SMD, 95\% CI 2.06, 1.36 to 2.77 ) at 12 months, with proximal and quadriceps rehabilitation compared to quadriceps rehabilitation alone.

\section{Mechanisms of proximal rehabilitation}

$\mathrm{Ten}^{24-26} 37394042 \quad 44-46$ of the 14 studies explored variables with potential to explain proximal rehabilitation effects in the short term. No studies investigated mechanisms of effect at medium or longer-term follow-up.

Proximal rehabilitation-compared with-control

Limited evidence (2 LQ study ${ }^{26}{ }^{46}$ ) of a large effect indicated that proximal rehabilitation using $\mathrm{OKC}$ band exercises increased isometric hip abduction strength $\left(\mathrm{I}^{2}=81 \%, \mathrm{p}<0.00001\right.$; $\mathrm{SMD}$,
95\% CI $1.69,1.03$ to 2.36 ) (figure 6). Very limited evidence (1 LQ study ${ }^{26}$ ) of a large effect indicated increased isometric hip external rotation strength in the $\mathrm{OKC}$ rehabilitation group in both the left (SMD, 95\% CI 2.45, 1.43 to 3.46) and right (SMD, 95\% CI 2.73, 1.66 to 3.80) hip. Very limited evidence (1 LQ study ${ }^{46}$ ) of a large effect indicated OKC proximal rehabilitation reduced the degree of knee valgum variability during consecutive footfalls while running (SMD, 95\% CI 2.68, 1.54 to 3.82 ), but did not change peak knee genu valgum angles while running (SMD, 95\% CI $0.83,-0.01$ to 1.67 ).

Proximal rehabilitation-compared with-quadriceps rehabilitation

Moderate evidence (1 HQ and 1 LQ study ${ }^{24}{ }^{42}$ ) indicated no difference in maximal isometric strength for hip abduction $\left(\mathrm{I}^{2}=60 \%, \mathrm{p}=0.11 ; \mathrm{SMD}, 95 \%\right.$ CI $0.18,-0.08$ to 0.44$)$, external rotation $\left(\mathrm{I}^{2}=0 \%, \mathrm{p}=0.44 ; \mathrm{SMD}, 95 \% \mathrm{CI} 0.11,-0.15\right.$ to 0.37 ) or knee extension $\left(\mathrm{I}^{2}=0 \%, \mathrm{p}=0.33\right.$; SMD, 95\% CI 0.09 , -0.17 to 0.35$)$ following a proximal $(\mathrm{OKC})$ rehabilitation programme, as compared to a quadriceps (OKC/CKC) rehabilitation programme (figure 7 ). Limited evidence (1 HQ study ${ }^{42}$ ) indicated no difference in hip extension (SMD, 95\% CI 0.09, -0.19 to 0.37 ) or internal rotation (SMD, 95\% CI 0.02, -0.26

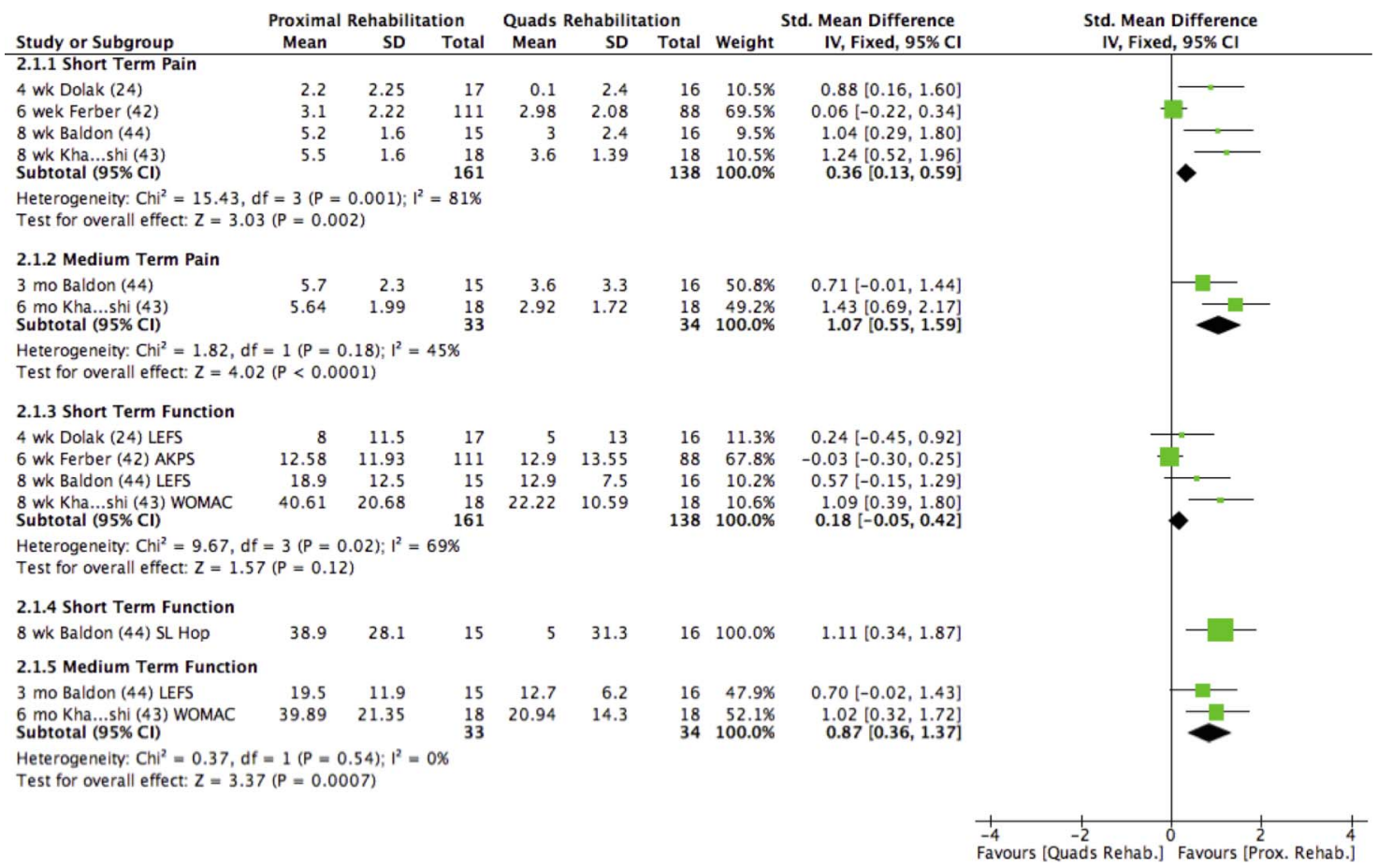

Figure 3 Proximal rehabilitation compared with quadriceps rehabilitation for pain and function (LEFS, Lower Extremity Functional Score; AKPS, Anterior Knee Pain Score; 'short term', <3 months; IV, inverse variance; Std., standard mean difference). 


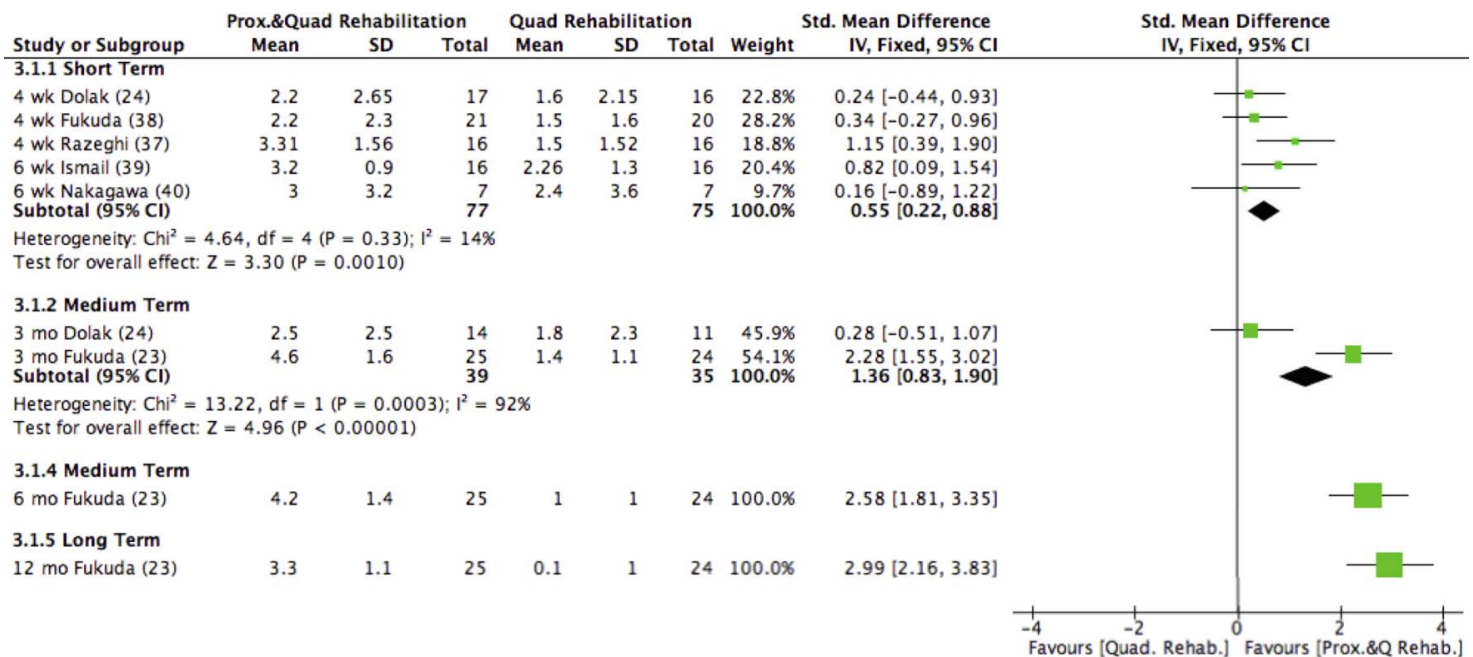

Figure 4 Proximal and quadriceps rehabilitation compared with quadriceps rehabilitation for pain ('short term', <3 months; 'medium term', 312 months; 'longer term', $\geq 12$ months; IV, inverse variance; Std., standard mean difference).

to 0.30 ) strength when comparing an OKC proximal with a CKC quadriceps rehabilitation programme.

Limited evidence ( $1 \mathrm{HQ}$ study ${ }^{44}$ ) indicated proximal rehabilitation using CKC exercises, when compared with a CKC quadriceps rehabilitation protocol, increased the degree of pelvis anteversion (SMD, 95\% CI 1.21, 0.43 to 1.98), hip flexion (SMD, 95\% CI 1.16, 0.39 to 1.92 ), trunk contralateral inclination (SMD, 95\% CI 0.90, 0.16 to 1.65 ), pelvis elevation (SMD, 95\% CI 0.94, 0.19 to 1.68), hip abduction (SMD, 95\% CI $2.20,1.28$ to 3.11 ), and knee adduction (SMD, 95\% CI $0.81,0.07$ to 1.55$)$ during a single leg squatting task. Additionally, anterior (SMD, 95\% CI 2.03, 1.14 to 2.92), lateral (SMD, 95\% CI 2.50, 1.53 to 3.46), and posterior (SMD, 95\% CI 1.54, 0.73 to 2.36) trunk endurance (seconds), and proximal abductor (SMD, 95\% CI 1.24, 0.47 to 2.02) and knee extensor (SMD, 95\% CI $0.97,0.22$ to 1.72$)$ torque $(\mathrm{nm} / \mathrm{kg}$ ) increased in the proximal rehabilitation group when compared with quadriceps rehabilitation. ${ }^{44}$

Proximal combined with quadriceps rehabilitation-compared with-quadriceps rehabilitation alone

Strong evidence ( $2 \mathrm{HQ}$ studies ${ }^{39}{ }^{40}$ ) indicated no difference in isokinetic, concentric and eccentric hip abduction, and external rotation strength following a proximal (OKC) combined with quadriceps (CKC) rehabilitation programme when compared to a quadriceps rehabilitation (CKC) programme alone (figure 8). Very limited evidence ( $1 \mathrm{LQ} \mathrm{study}^{24}$ ) indicated no difference in isometric hip abduction (SMD, 95\% CI $0.54,-0.16$ to 1.23 ) or external rotation (SMD, 95\% CI $0.63,-0.07$ to 1.33 ) strength following a proximal combined with quadriceps rehabilitation

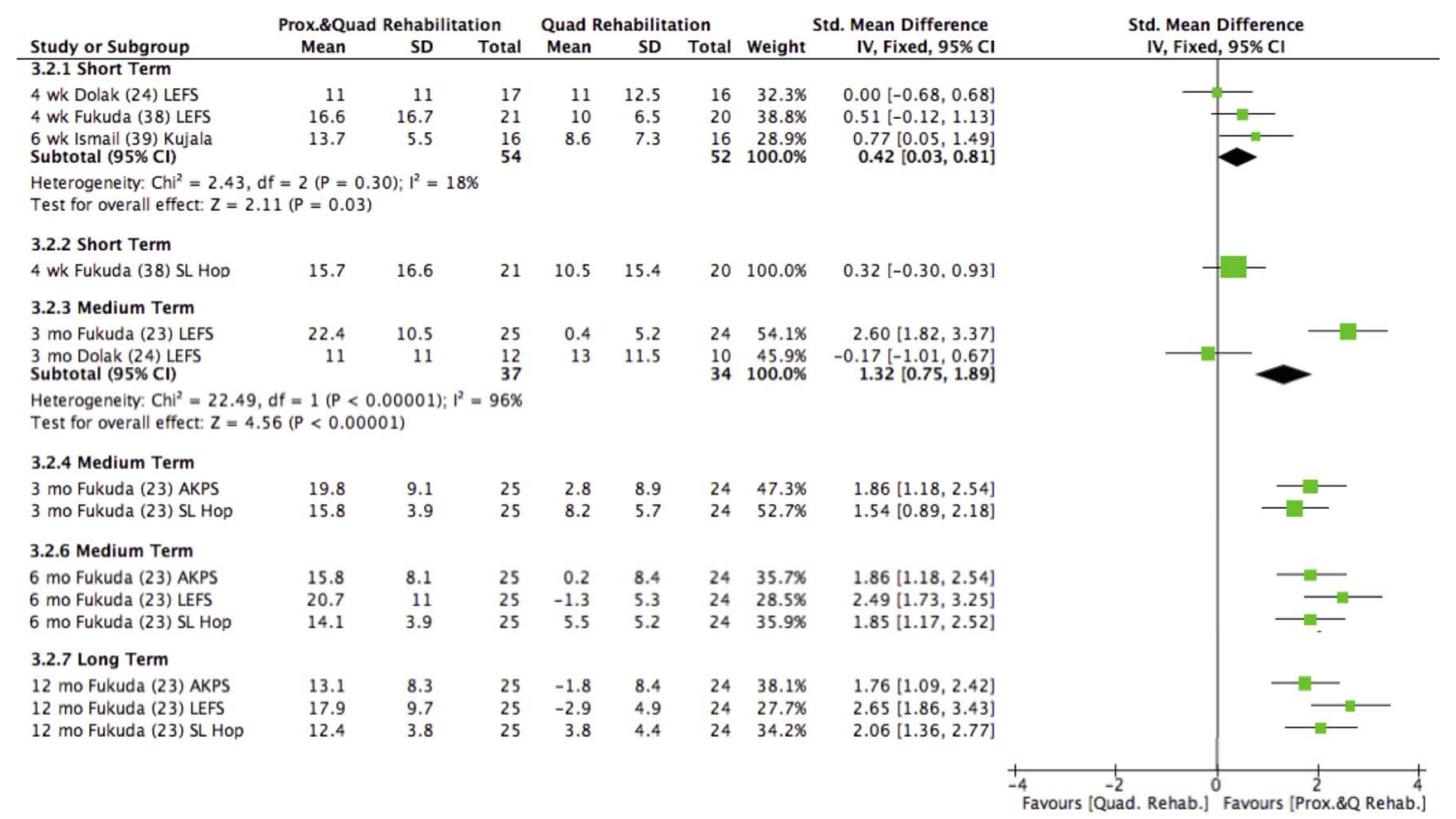

Figure 5 Proximal and quadriceps rehabilitation compared with quadriceps rehabilitation for function (LEFS, Lower Extremity Functional Score; AKPS, Anterior Knee Pain Score; 'short term', <3 months; 'medium term', 3-12 months; 'longer term', $\geq 12$ months; IV, inverse variance; Std., standard mean difference). 


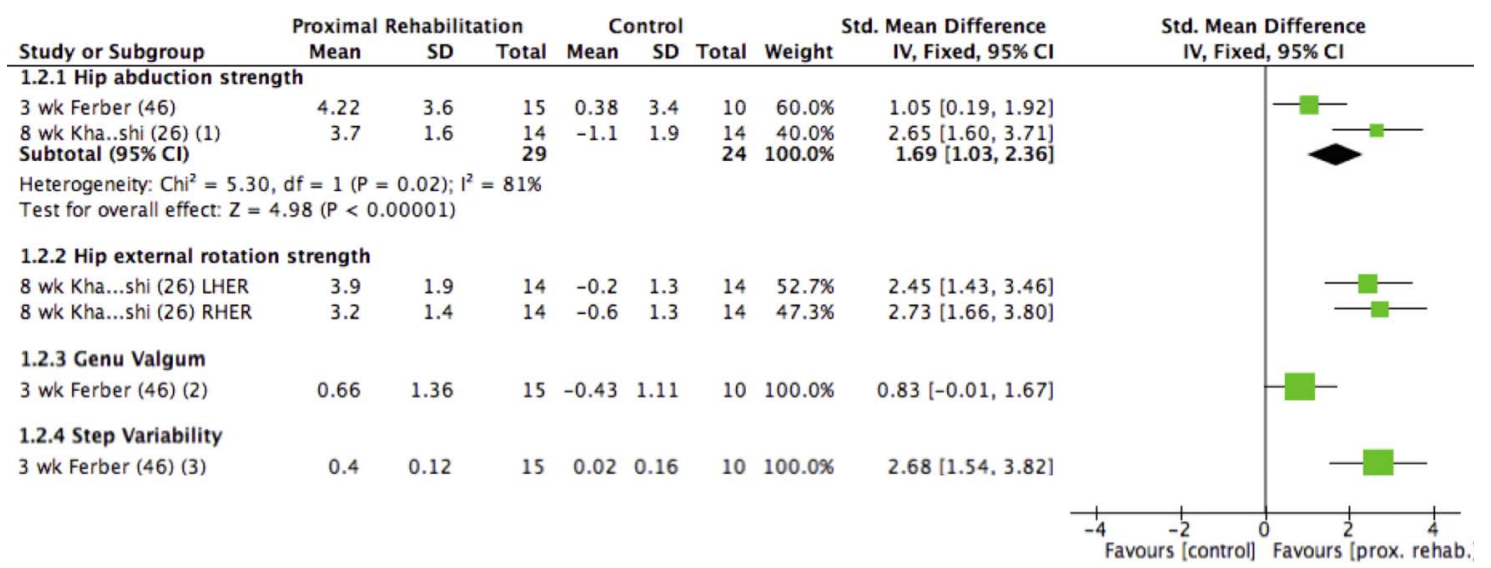

(1) Right leg data used for pooling

(2) Degrees

(3) Consecutive footfall variability

Figure 6 Proximal rehabilitation compared with control group for strength and biomechanics in the short term ( $<3$ months) (LHER, left proximal external rotation; RHER, right proximal external rotation; IV, inverse variance; Std., standard mean difference).

programme compared to quadriceps rehabilitation alone. Moderate evidence ( 2 LQ studies ${ }^{24}$ ) ) indicated no difference in isometric knee extension strength $\left(\mathrm{I}^{2}=0 \%, \mathrm{p}=0.37\right.$; SMD, $95 \%$ CI $0.07,-0.42$ to 0.56 ) following comparison of proximal and quadriceps rehabilitation with quadriceps rehabilitation alone.

\section{Proximal rehabilitation alone}

One LQ study ${ }^{45}$ divided their cohort into 'successful' $(\geq 1.5 \mathrm{~cm}$ reduction in VAS) and 'unsuccessful' groups, reporting an improvement in isometric proximal abduction and adduction strength was unrelated to success. Very limited evidence (1 LQ study ${ }^{25}$ ) indicated an improvement in lateral core endurance $(p=0.001)$, hip abduction $(p=0.008)$ and external rotation $(p=0.03)$ isometric strength, and knee abduction internal moments $(\mathrm{p}=0.05)$ after an 8-week OKC and CKC neuromuscular activation intervention directed at the proximal musculature. A trend in increased anterior $(p=0.06)$ and posterior $(\mathrm{p}=0.1)$ core endurance, and hip abduction moment $(\mathrm{p}=0.06)$ was reported in the same study. ${ }^{25}$ However, no change in rear foot eversion, knee abduction, hip adduction and internal rotation angles, rear foot inversion or hip external rotation moments were reported during a running task. ${ }^{25}$

\section{DISCUSSION}

This systematic review and meta-analysis evaluated the effects of proximal muscle rehabilitation on pain and function in individuals with PFP and the potential mechanisms for effectiveness. Fourteen studies of varying quality were identified, including 11 RCTs. $^{23} 2426{ }^{37-44}$ In the short term, strong evidence indicates proximal combined with quadriceps rehabilitation is significantly better at reducing pain than quadriceps rehabilitation alone, ${ }^{23} 24 \quad 37 \quad 39-41 \quad 46$ moderate evidence indicates proximal rehabilitation is better at improving pain compared to quadriceps rehabilitation alone, ${ }^{24} 42-44$ and very limited evidence indicates proximal rehabilitation reduces pain compared to a no

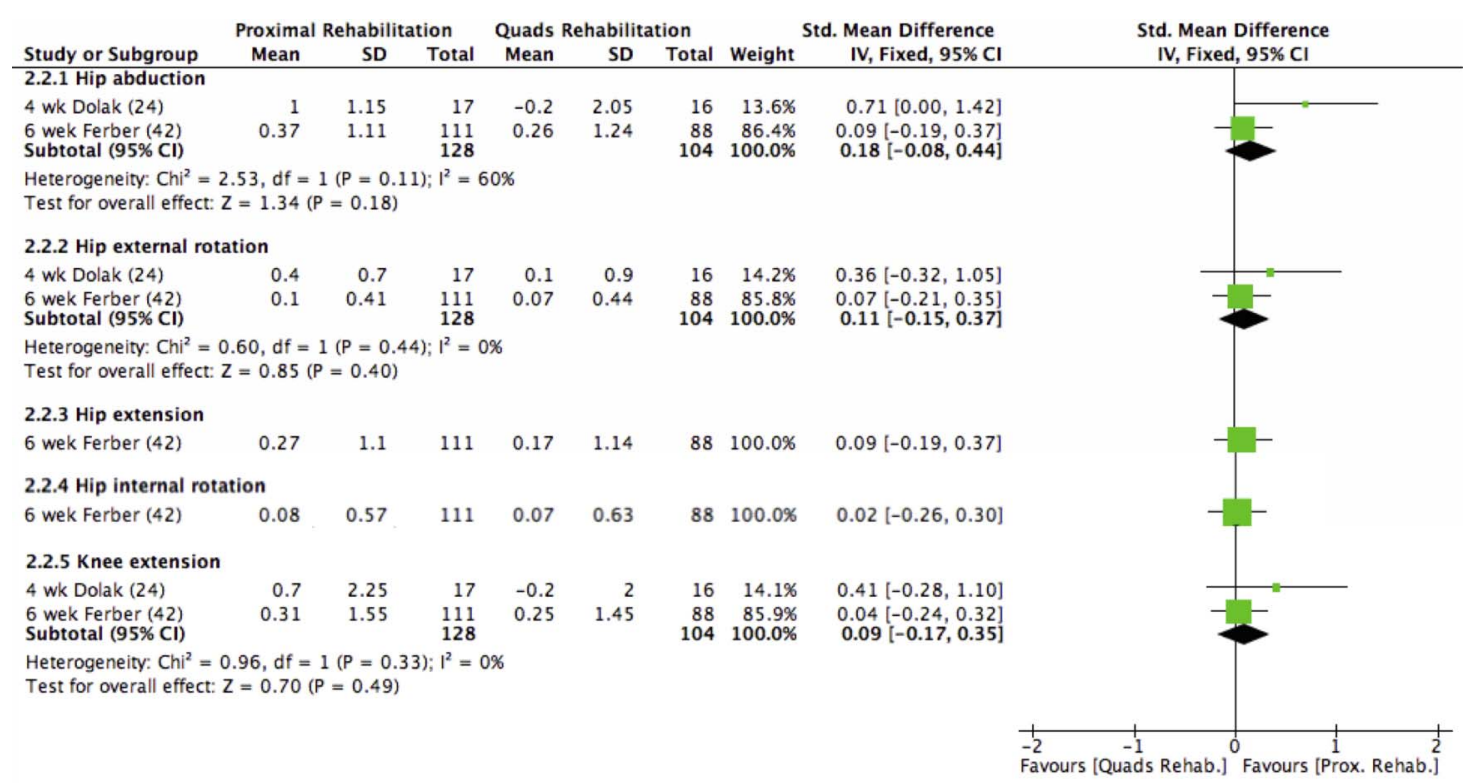

Figure 7 Proximal rehabilitation compared with quadriceps rehabilitation for isometric strength in the short term ( $<3$ months) (IV, inverse variance; Std., standard mean difference). 


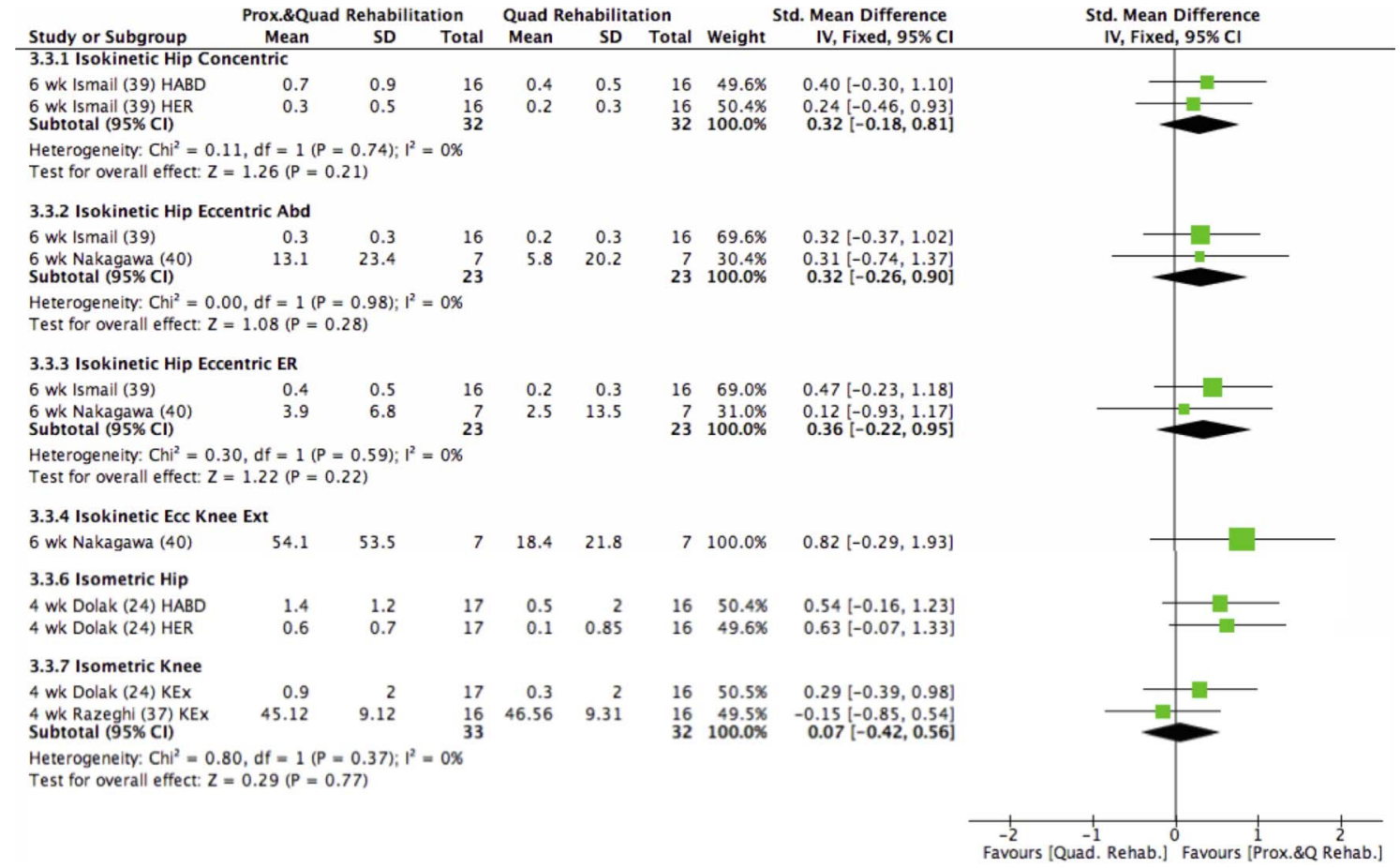

Figure 8 Proximal and quadriceps rehabilitation compared with quadriceps rehabilitation for strength in the short term ( $<3$ months) (Abd, abduction; ER, external rotation; Ecc, eccentric; Ext, extension; HABD, hip abduction; HER, hip external rotation; IV, Inverse variance; Std., standard mean difference.

intervention control. ${ }^{26}$ In the medium term, strong and moderate evidence indicates proximal and proximal combined with quadriceps rehabilitation, respectively, is more effective at reducing pain then quadriceps rehabilitation alone. ${ }^{23} 4344$ In the longer term, limited evidence indicates proximal combined with quadriceps rehabilitation is more effective at reducing pain than quadriceps rehabilitation alone. ${ }^{23}$ Greater improvements in function were also reported for proximal, and proximal combined with quadriceps rehabilitation compared with quadriceps rehabilitation alone in the short (strong evidence), medium (strong to moderate evidence) and longer (limited evidence) term. ${ }^{23} 243839$ Put together, these findings support the implementation of proximal muscle rehabilitation programmes for the management of PFP in clinical practice.

\section{Effects of proximal rehabilitation}

\section{Pain}

Strong evidence suggests that a combined proximal and quadriceps rehabilitation protocol using both $\mathrm{OKC}$ and CKC exercises results in superior short term outcomes of pain reduction when compared to CKC quadriceps rehabilitation alone. This pooled result is, however, driven by one LQ study ${ }^{37}$ that reported significant improvement in pain. In contrast, three 3840 of the five pooled studies reported no greater short-term pain reduction when compared to a quadriceps rehabilitation programme. An important consideration when interpreting these findings is that the CKC quadriceps exercises adopted within the three equivocal studies 2438 would also facilitate activation of proximal musculature and as such, could also be considered a combined proximal and quadriceps intervention. A similar story emerges when comparing proximal and quadriceps rehabilitation. Specifically, within a pooled group of heterogeneous studies that evaluated proximal compared with quadriceps rehabilitation, a large multicentre trial that compared an OKC proximal to a CKC quadriceps protocol demonstrated no significant differences in pain reduction between groups, but reported significant pain reduction within both groups in the short term. ${ }^{42}$ Adding proximal rehabilitation to quadriceps appears to offer the most favourable short-term outcome; however, it is clear that further research to identify the most effective rehabilitation programme design is required to maximise effectiveness.

In the medium term, pooled results indicate proximal rehabilitation more effectively reduced pain when compared to quadriceps rehabilitation alone. Among the pooled studies, exercise programme design differed significantly, with one utilising exclusively neuromuscular $\mathrm{OKC}^{43}$ and the other using neuromuscular and strength $\mathrm{CKC}^{44}$ exercises. Of these protocols, the neuromuscular OKC protocol resulted in the greatest positive symptom change, but further research directly comparing these different protocols is needed. Proximal combined with quadriceps rehabilitation was more effective than quadriceps rehabilitation in reducing pain in the medium and longer term, with treatment effects of large magnitude. The single study reporting significant pain reduction in the long-term used a combination of both OKC and CKC at an intensity assessed to be sufficient to evoke strength changes (ie, $>70 \% 1 \mathrm{RM}$ ). ${ }^{23}$ These exercise parameters may be relevant to the successful outcome given that OKC exercises are commonly used clinically to allow for specific isolation of proximal musculature ${ }^{48}$ while attempting to reduce loading of the PFJ. These preliminary findings require further research to determine the most efficacious protocol to reduce pain, in the medium and long term.

\section{Function}

Fewer studies evaluated the effects of proximal muscle rehabilitation on functional outcomes, which limits the conclusions that can be drawn. Greater improvements in self-reported measures (WOMAC, LEFS, AKPS) and performance-based measures (Single Leg hop test) of function were observed in the short 
term following proximal rehabilitation compared to a no intervention control; and following proximal combined with quadriceps rehabilitation compared to quadriceps rehabilitation. Moderate evidence indicated no difference in function when comparing proximal with quadriceps rehabilitation, which differs from findings related to pain. It is possible that interventions that address the deficits in gluteal strength ${ }^{20} 19$ and activation $^{21}$ and quadriceps strength ${ }^{19}$ that are evident in individuals with PFP could be sufficient to result in the short-term improvement of function, but not in the medium and longer term. Importantly, greater functional improvements are provided by proximal compared to quadriceps rehabilitation (strong evidence), and a combined proximal and quadriceps rehabilitation compared to quadriceps rehabilitation alone (limited to moderate evidence) in the medium and longer term. It is possible that superior pain outcomes with proximal compared to quadriceps rehabilitation in the short-term results from the quadriceps loading causing irritation to the PFJ, although further research documenting pain levels during exercise is needed to clarify this. These findings suggest that incorporating proximal rehabilitation into the management of PFP is beneficial to functional outcomes. However, considering the currently limited to moderate supporting evidence, further high quality studies are needed to confirm these positive results.

\section{Mechanisms of proximal rehabilitation}

This review evaluated potential short-term mechanisms of treatment effect, with studies reporting improvement in isometric $^{242646}$ and isokinetic strength, ${ }^{39}{ }^{40}$ eccentric torque, ${ }^{44}$ trunk muscle endurance, ${ }^{44}$ single leg squat kinematics, ${ }^{44}$ reduced knee joint motion variability, ${ }^{46}$ and reduced peak knee abduction internal moments. ${ }^{25}$ Changes in strength were measured in 10 of the 14 included studies. Limited evidence of large effect indicated greater improvement in isometric hip abduction strength in the short-term following a neuromuscular or strengthendurance resistance band intervention when compared to no intervention, offering a potential mechanism for changes in pain and function. ${ }^{26}{ }^{46}$ However, given the absence of significant difference in maximal isometric hip abduction, extension, external and internal rotation, and knee extension strength when comparing OKC proximal rehabilitation to CKC quadriceps rehabilitation, ${ }^{42}$ it is also plausible that the positive effects of a rehabilitation intervention are not exclusively derived through changes in strength, but through a combination of more global lower limb strength changes, a change in lower limb biomechanics, possible central or systemic mechanisms, ${ }^{49}$ or most likely a combination of these factors.

Three studies exploring kinematic and/or kinetic change resulting from proximal rehabilitation reported small but significant changes about the hip and knee $e^{25} 44$ that offer potential mechanisms for treatment effects. Increased hip adduction and internal rotation has been identified previously as a risk factor for and is associated with PFP symptoms. ${ }^{5} 14$ Consequently interventions that demonstrate capacity to modify these parameters warrant further investigation. Although some evidence reports clinical measures are able to identify individuals with movement deficits and detect biomechanical change of small magnitude, ${ }^{50}$ it is important to ensure clinical tools are sufficiently sensitive to direct intervention choice and determine treatment effects.

Further exploration of the mechanisms for treatment effects following a rehabilitation intervention on muscle structure, neural innervation, systemic systems and biomechanics is needed to guide a tailored exercise approach to address patient-specific deficits and predict outcomes in individuals with PFP.

\section{Clinical implications}

Interventions that aim to improve strength, strength-endurance and neuromuscular activity of proximal musculature are effective in the management of PFP, and should be incorporated in clinical practice. From within the largest high quality study that explored the effect of proximal compared with quadriceps rehabilitation, OKC proximal rehabilitation in isolation was not more effective at reducing pain than a CKC quadriceps rehabilitation programme ${ }^{42}$ in the short term. These findings suggest CKC quadriceps rehabilitation is as effective at increasing proximal strength as an isolated OKC proximal rehabilitation programme and have potentially important clinical implications for exercise intervention design. These suggest that clinicians could choose different treatment approaches and achieve the same or similar strength gains. Subsequently, this would allow clinicians to be guided by patient response, preference or available equipment, without negatively impacting on patient care.

Proximal rehabilitation combined with quadriceps rehabilitation using both $\mathrm{OKC}$ and $\mathrm{CKC}$ exercises produced better pain and functional outcomes in the short, ${ }^{44}$ medium and longer term $^{23}$ compared to quadriceps rehabilitation alone. Therefore, therapists treating PFP should aim to prescribe exercise interventions targeting the quadriceps and proximal musculature in individuals with PFP using a combination of $\mathrm{OKC}$ and $\mathrm{CKC}$ exercises.

Rehabilitation exercises from studies included in this review were completed between 3 and 7 times per week, with the intensity of the programmes varying significantly. Exercise frequency did not appear to be dependent on the type and intensity of the rehabilitation protocols prescribed. Given established guidelines $^{33}$ that indicate neuromuscular training has greater effect if performed frequently (daily) and strength training less frequently $(2-3 \times$ per week) on physiological adaptation, the specificity of exercise frequency in the management of PFP is lacking within the current evidence base and requires further research.

Given that the primary goal of PFP treatment is often pain reduction, utilising exercise parameters that do not aggravate symptoms is important. Consequently, utilising OKC exercises in the short term or CKC exercises within finite pain limits throughout the rehabilitation process is advocated by the current evidence. ${ }^{23} 24{ }^{44}$ It is unclear at present whether these parameters are essential for a successful outcome; further research is required to determine the most effective protocol design. Furthermore, identification of individuals who are more likely to respond to a specific proximal intervention approach is an important consideration given the dearth of evidence exploring indicators for proximal intervention success. ${ }^{51}$

\section{Limitations}

There are limitations that need to be considered when interpreting the results of this review. Variability in study design, type of protocol (OKC or $\mathrm{CKC}$ ), and differing outcome measures limited further data pooling. Where pooling was possible, heterogeneity of rehabilitative exercise prescription remained evident in both exercise frequency and intensity. Nonetheless, given the paucity of available evidence, we felt that data pooling was valuable to strengthening the findings of the review. Non-English language publications were not sought in this review. While it is arguable that high-quality RCTs would aim to be published in higher impact journals written in the English language, identification of further trial data may have influenced the outcomes of analysis. Assessment of methodological quality 
was completed using the PEDro scale. It was identified that only $43 \%$ of the included 11 studies blinded the assessor to the intervention delivered and only one study attempted to blind the participants to their group allocation. Given growing evidence for proximal intervention efficacy, future studies should aim to blind the participants and researchers to the allocated group. Finally, it has been identified that mechanobiological determinants of exercise prescription are poorly reported or defined within the included rehabilitation studies. To ensure clinical utility and consistency of exercise parameter reporting within future studies, these determinants must be detailed.

\section{CONCLUSION}

The best available evidence indicates that proximal rehabilitation with or without simultaneous quadriceps rehabilitation is beneficial to pain and function in individuals with PFP in the short and medium term. While fewer studies have evaluated long-term effects, the limited evidence available indicates proximal and quadriceps rehabilitation combined has greater positive benefit on pain and function than quadriceps rehabilitation alone. A combination of both $\mathrm{OKC}$ and CKC exercise are most likely to result in favourable outcomes. Given variability in rehabilitation protocols within the current literature, further studies designed to identify the most effective protocol by considering exercise type, load and dose are required. To improve clinical applicability, these studies must detail specific exercise descriptors. Regardless, proximal rehabilitation should be incorporated into clinical reasoning paradigms for the management of PFP.

\section{Summary box}

- Proximal combined with quadriceps rehabilitation reduces pain and improves function more than quadriceps rehabilitation in the short, medium and long term.

- Proximal rehabilitation is more effective than quadriceps rehabilitation in decreasing pain in the short term, and decreasing pain and improving function in the medium term.

- Maximum hip muscle isometric strength can be increased by proximal rehabilitation compared to no exercise controls, but not significantly more than a closed kinetic chain quadriceps focused rehabilitation programme.

Funding \#TEAM_PFP would like to acknowledge the Private Physiotherapy Education Fund (PPEF) for funding some of SL's time to complete this review. DM is part funded by the NIHR/HEE Senior Clinical Lecturer scheme. This article presents independent research part funded by the National Institute for Health Research (NIHR). The views expressed are those of the authors and not necessarily those of the NHS, the NIHR or the Department of Health.

Competing interests None declared.

Provenance and peer review Not commissioned; externally peer reviewed.

\section{REFERENCES}

1 van Middelkoop M, van Linschoten R, Berger MY, et al. Knee complaints seen in general practice: active sport participants versus non-sport participants. $B M C$ Musculoskelet Disord 2008;9:36.

2 Baquie P, Brukner P. Injuries presenting to an Australian sports medicine centre: a 12-month study. Clin J Sport Med 1997;7:28-31.

3 Boling M, Padua D, Marshall S, et al. Gender differences in the incidence and prevalence of patellofemoral pain syndrome. Scand I Med Sci Sports 2010;20:725-30.

4 Thijs $Y$, Pattyn E, Van Tiggelen D, et al. Is hip muscle weakness a predisposing factor for patellofemoral pain in female novice runners? A prospective study. Am J Sports Med 2011;39:1877-82.

5 Noehren B, Hamill J, Davis I. Prospective evidence for a hip etiology in patellofemoral pain. Med Sci Sports Exerc 2013;45:1120-4.
6 Heino Brechter J, Powers CM. Patellofemoral stress during walking in persons with and without patellofemoral pain. Med Sci Sports Exerc 2002;34: 1582-93.

7 Farrokhi S, Keyak JH, Powers CM. Individuals with patellofemoral pain exhibit greater patellofemoral joint stress: a finite element analysis study. Osteoarthritis Cartilage 2011;19:287-94.

8 Draper CE, Besier TF, Santos JM, et al. Using real-time MRI to quantify altered joint kinematics in subjects with patellofemoral pain and to evaluate the effects of a patellar brace or sleeve on joint motion. J Orthop Res 2009:27:571-7.

9 Wilson NA, Press JM, Koh JL, et al. In vivo noninvasive evaluation of abnormal patellar tracking during squatting in patients with patellofemoral pain. J Bone Joint Surg Am 2009;91:558-66.

10 Souza RB, Draper CE, Fredericson M, et al. Femur rotation and patellofemoral joint kinematics: a weight-bearing magnetic resonance imaging analysis. J Orthop Sports Phys Ther 2010;40:277-85.

11 Giles LS, Webster KE, McClelland JA, et al. Does quadriceps atrophy exist in individuals with patellofemoral pain? A systematic literature review with meta-analysis. J Orthop Sports Phys Ther 2013;43:766-76.

12 Barton CJ, Levinger P, Crossley KM, et al. Relationships between the Foot Posture Index and foot kinematics during gait in individuals with and without patellofemoral pain syndrome. J Foot Ankle Res 2011;4:10.

13 Noehren $B$, Pohl MB, Sanchez $Z$, et al. Proximal and distal kinematics in female runners with patellofemoral pain. Clin Biomech 2012;27:366-71.

14 Powers CM. The influence of abnormal hip mechanics on knee injury: a biomechanical perspective. J Orthop Sports Phys Ther 2010;40:42-51.

15 Witvrouw E, Callaghan MJ, Stefanik JJ, et al. Patellofemoral pain: consensus statement from the 3rd International Patellofemoral Pain Research Retreat held in Vancouver, September 2013. Br J Sports Med 2014;48: 411-14.

16 Collins NJ, Bisset LM, Crossley KM, et al. Efficacy of nonsurgical interventions for anterior knee pain: systematic review and meta-analysis of randomized trials. Sports Med 2012;42:31-49.

17 Barton CL, Lack S, Hemmings S, et al. The 'Best Practice Guide to Conservative Management of Patellofemoral Pain' - incorporating level 1 evidence with expert clinical reasoning. Br J Sports Med 2015;49:923-34

18 Prins MR, van der Wurff P. Females with patellofemoral pain syndrome have weak hip muscles: a systematic review. Aust J Phys 2009;55:9-15.

19 Lankhorst NE, Bierma-Zeinstra SM, van Middelkoop M. Factors associated with patellofemoral pain syndrome: a systematic review. $\mathrm{Br}$ J Sports Med 2013:47:193-206.

20 Rathleff MS, Rathleff CR, Crossley KM, et al. Is hip strength a risk factor for patellofemoral pain? A systematic review and meta-analysis. Br J Sports Med 2014:48:1088.

21 Barton CJ, Lack S, Malliaras $P$, et al. Gluteal muscle activity and patellofemoral pain syndrome: a systematic review. Br J Sports Med 2013;47:207-14.

22 Boling MC, Padua DA, Marshall SW, et al. A prospective investigation of biomechanical risk factors for patellofemoral pain syndrome the joint undertaking to monitor and prevent ACL injury (JUMP-ACL) cohort. Am J Sports Med 2009:37:2108-16.

23 Fukuda TY, Melo WP, Zaffalon BM, et al. Hip posterolateral musculature strengthening in sedentary women with patellofemoral pain syndrome: a randomized controlled clinical trial with 1-year follow-up. J Orthop Sports Phys Ther 2012;42:823-30.

24 Dolak KL, Silkman C, Medina McKeon J, et al. Hip strengthening prior to functional exercises reduces pain sooner than quadriceps strengthening in females with patellofemoral pain syndrome: a randomized clinical trial. J Orthop Sports Phys Ther 2011;41:560-70.

25 Earl JE, Hoch AZ. A proximal strengthening program improves pain, function, and biomechanics in women with patellofemoral pain syndrome. Am J Sports Med 2011;39:154-63.

26 Khayambashi K, Mohammadkhani Z, Ghaznavi K, et al. The effects of isolated hip abductor and external rotator muscle strengthening on pain, health status, and hip strength in females with patellofemoral pain: a randomized controlled trial. J Orthop Sports Phys Ther 2012;42:22-9.

27 Barton C, Hemmings S, Morrissey D. The influence of published evidence on physiotherapists' clinical reasoning when treating patellofemoral pain syndrome: a mixed-methods study. J Orthop Sports Phys Ther 2012;42:A49-50.

28 Peters JS, Tyson NL. Proximal exercises are effective in treating patellofemoral pain syndrome: a systematic review. Int J Sports Phys Ther 2013;8:689-700.

29 Moher D, Liberati A, Tetzlaff J, et al. Preferred reporting items for systematic reviews and meta-analyses: the PRISMA statement. BMJ 2009;339:b2535.

30 Verhagen AP, de Vet HC, de Bie RA, et al. The Delphi list: a criteria list for quality assessment of randomized clinical trials for conducting systematic reviews developed by Delphi consensus. J Clin Epidemiol 1998;51:1235-41.

31 Barton CJ, Webster KE, Menz HB. Evaluation of the scope and quality of systematic reviews on nonpharmacological conservative treatment for patellofemoral pain syndrome. J Orthop Sports Phys Ther 2008;38:529-41. 
32 Moher D, Jadad AR, Tugwell P. Assessing the quality of randomized controlled trials. Current issues and future directions. Int I Technol Assess Health Care 1996;12:195-208.

33 American College of Sports M. American College of Sports Medicine position stand. Progression models in resistance training for healthy adults. Med Sci Sports Exerc 2009;41:687-708.

34 Hume P, Hopkins W, Rome K, et al. Effectiveness of foot orthoses for treatment and prevention of lower limb injuries. Sports Med 2008:38:759-79.

35 Cohen J. A power primer. Psychol Bull 1992;112:155-9.

36 Van Tulder M, Furlan A, Bombardier C, et al. Group EBotCCBR. Updated method guidelines for systematic reviews in the Cochrane Collaboration Back Review Group. Spine 2003:28:1290-9.

37 Razeghi M, Etemadi Y, Taghizadeh S, et al. Could hip and knee muscle strengthening alter the pain intensity in patellofemoral pain syndrome? IRCMJ 2010;12:104-10.

38 Fukuda TY, Rossetto FM, Magalhaes E, et al. Short-term effects of hip abductors and lateral rotators strengthening in females with patellofemoral pain syndrome: a randomized controlled clinical trial. J Orthop Sports Phys Ther 2010;40:736-42.

39 Ismail MM, Gamaleldein MH, Hassa KA. Closed kinetic chain exercises with or without additional hip strengthening exercises in management of patellofemoral pain syndrome: a randomized controlled trial. Eur J Phys Rehabil Med 2013;49:687-98

40 Nakagawa TH, Muniz TB, Baldon RdM, et al. The effect of additional strengthening of hip abductor and lateral rotator muscles in patellofemoral pain syndrome: a randomized controlled pilot study. Clin Rehabil 2008;22:1051-60.

41 Avraham $F$, Aviv $S$, Ya'akobi $P$, et al. The efficacy of treatment of different intervention programs for patellofemoral pain Syndrome-A single blinded randomized clinical trial. Pilot study. The Scientific World Journal 2007;7:1256-62.
42 Ferber R, Bolgla L, Earl-Boehm JE, et al. Strengthening of the hip and core versus knee muscles for the treatment of patellofemoral pain: a multicenter, randomized controlled trial. J Athl Train 2015;50:366-77.

43 Khayambashi K, Fallah A, Movahedi A, et al. Posterolateral hip muscle strengthening versus quadriceps strengthening for patellofemoral pain: a comparative control trial. Arch Phys Med Rehabil 2014;95:900-7.

44 Baldon RDM, Serrão FV, Scattone Silva R, et al. Effects of functional stabilization training on pain, function, and lower extremity biomechanics in women with patellofemoral pain: a randomized clinical trial. I Orthop Sports Phys Ther 2014:44:240-A8.

45 Tyler TF, Nicholas SJ, Mullaney MJ, et al. The role of hip muscle function in the treatment of patellofemoral pain syndrome. Am J Sports Med 2006;34:630-7.

46 Ferber $R$, Kendall $D$, Farr L. Changes in knee biomechanics after a hip-abductor strengthening protocol for runners with patellofemoral pain syndrome. J Athl Train 2011:46:142-50

47 Toigo $\mathrm{M}$, Boutellier U. New fundamental resistance exercise determinants of molecular and cellular muscle adaptations. Eur J Appl Physiol 2006;97:643-63.

48 Selkowitz DM, Beneck GJ, Powers CM. Which exercises target the gluteal muscles while minimizing activation of the Tensor Fascia Lata? Electromyographic assessment using fine-wire electrodes. J Orthop Sports Phys Ther 2013;43:54-64.

49 Turner A, Comfort P, Moody J, et al. Neuroendocrinology and resistance training in adult males. Prof Strength Cond 2010;17:15-24.

50 Whatman $C$, Hume $P$, Hing W. The reliability and validity of physiotherapist visual rating of dynamic pelvis and knee alignment in young athletes. Phys Ther Sport 2013;14:168-74

51 Lack S, Barton C, Vicenzino B, et al. Outcome predictors for conservative patellofemoral pain management: a systematic review and meta-analysis. Sports Med 2014;44:1703-16. 